\title{
Aspectos clínicos e nutricionais em pessoas vivendo com HIV/AIDS: uma série de casos
}

\author{
Clinical and nutritional aspects in people living with HIV/AIDS: a case series
}

Aspectos clínicos y nutricionales en personas viviendo con VIH/SIDA: una serie de casos

Isabel Oliveira Aires ${ }^{1 *}$, Nayra do Socorro Caldas Carvalho de Almeida Teixeira1, lara Katrynne Fonseca Oliveira ${ }^{1}$, Renata Rios Torres Rodrigues'1, Robson Eduardo da Silva Araújo', Dorcas Lamounier Costa ${ }^{1}$, Cecília Maria Resende Gonçalves de Carvalho ${ }^{1}$, Adriana de Azevedo Paiva ${ }^{1}$

\section{RESUMO}

Objetivo: Descrever, em formato de série de casos, aspectos clínicos e nutricionais de pessoas que vivem com HIV/AIDS(PVHA) atendidas em ambulatório de hospital de referência do Nordeste. Detalhamento dos casos: Os dados clínicos foram obtidos em prontuários médicos; o estado nutricional avaliado pelo índice de massa corporal, circunferência da cintura e circunferência do pescoço; e o consumo alimentar pela análise do recordatório de 24h. Foram descritos 8 casos de PVHA de ambos os sexos, maiores de 20 anos, em uso de terapia antirretroviral(TARV). Os pacientes estavam há pelo menos 1 mês em uso de TARV. Predominouse o uso de Lamivudina e Tenofovir, ambos inibidores nucleosídeos da transcriptase reversa, e o Dolutegravir, inibidor de protease, e seu uso está associado ao surgimento de sintomas gastrintestinais, como náuseas, vômitos e constipação. O estado nutricional mostrou que, decorrente do avanço da TARV, os casos de desnutrição em PVHA declinou, dando lugar à eutrofia e excesso de peso. Considerações finais: Os relatos reforçam a importância do monitoramento não apenas dos aspectos clínicos, mas também dos aspectos nutricionais, aspirando alcançar a assistência integral e individualizada para as PVHA, bem como indicam a pertinência de se realizar estudos mais abrangentes acerca dos aspectos nutricionais em PVHA.

Palavras-chave: Saúde Pública, HIV, Estado Nutricional, Relatos de casos.

\begin{abstract}
Objective: To describe, in a series of cases, clinical and nutritional aspects of people living with HIV/AIDS (PLWHA) attended at an outpatient clinic of a reference hospital in the northeast region of Brazil. Details of the cases: Clinical data were obtained from medical records; nutritional status assessed by body mass index, waist circumference and neck circumference; and the food consumption by the analysis of a 24-hour recall. Were described 8 cases of PLWHA of both sexes, older than 20 years, using antiretroviral therapy (ART). The patients were at least 1 month on ART. Were used predominantly Lamivudine and Tenofovir, both nucleoside reverse transcriptase inhibitors, and Dolutegravir, a protease inhibitor, witch its use is associated with gastrointestinal symptoms, such as nausea, vomiting and constipation. The nutritional status showed that, as a result of ART progress, the cases of PLWHA malnutrition declined, leading to eutrophy and overweight. Final considerations: The reports reinforce the importance of monitoring not only the clinical aspects, but also the nutritional aspects, aspiring to achieve integral and individualized care for PLWHA, as well as indicate the relevance of carrying out more comprehensive studies on nutritional aspects in PLWHA.
\end{abstract}

Keywords: Public Health, VIH, Nutritional Status, Case Reports.

1Universidade Federal do Piauí, Teresina-Piauí. *E-mail: i-aires@hotmail.com

Financiamento: Fundação de Amparo à Pesquisa do Estado do Piauí - FAPEPI - EDITAL FAPEPI/MSDECIT/CNPq/SESAPI No 002/2016 - PPSUS - Processo 027/2016

SUBMETIDO EM: 6/2019 | ACEITO EM: 7/2019 | PUBLICADO EM: 7/2019

REAS | Vol. Sup.28 | e1077 | DOI: https://doi.org/10.25248/reas.e1077.2019 Página 1 de 6 


\section{RESUMÉN}

Objetivo: Describir, en formato de serie de casos, aspectos clínicos y nutricionales en personas que viven con HIV/AIDS (PVHA) atendidas en ambulatorio de hospital de referencia del Nordeste. Método: Los datos clínicos se obtuvieron en historias médicas; el estado nutricional (EN) evaluado por el índice de masa corporal, circunferencia de la cintura y circunferencia del cuello; y el consumo energético alimentario por el análisis del recordatorio de las 24h. Detalle de los casos: se describieron 8 casos de PVHA de ambos sexos, mayores de 20 años, en uso de terapia antirretroviral (TARV). Los pacientes tenían al menos 1 mes en el uso de TARV. Se utilizó el uso de Lamivudina y Tenofovir, ambos inhibidores nucleósidos de la transcriptasa inversa, y el Dolutegravir, inhibidor de proteasa, y su uso está asociado al surgimiento de síntomas gastrointestinales, como náuseas, vómitos y constipación. El estado nutricional mostró que, gracias al avance de la TARV, los casos de desnutrición en PVHA declinó, dando lugar a la eutrofia y sobrepeso. Consideraciones finales: Los relatos refuerzan la importancia del monitoreo no sólo de los aspectos clínicos, sino también de los aspectos nutricionales, aspirando a alcanzar la asistencia integral e individualizada para las PVHA, así como, indican la pertinencia de realizar estudios más amplios sobre los aspectos nutricionales en PVHA.

Palabras clave: Salud Pública, HIV, estado nutricional, informes de casos.

\section{INTRODUÇÃO}

Desde o século XI, o HIV e a Aids representam um dos maiores problemas de saúde pública, devido sua gravidade e seu caráter pandêmico (BRASIL, 2013).

Em 2016, cerca de 36,7 milhões de pessoas viviam com HIV, foram notificados 1,8 milhões de novos casos de infecção pelo vírus e registradas aproximadamente 1 milhão de mortes por causas relacionadas ao HIV no mundo (OMS, 2017).

No mesmo ano, no Brasil, foram registradas 38 mil novas infecções, e entre 2006 e 2016 houve um aumento na taxa de detecção do vírus (BRASIL, 2017).

A terapia antirretroviral (TARV) é distribuída gratuitamente no Brasil desde 1996, e tal tratamento possibilita a redução da morbimortalidade da doença, além de aumentar o tempo de sobrevida de pessoas que vivem com HIV/Aids (PVHA). Seu objetivo é estabilizar a carga viral plasmática ao nível inferior a 50 cópias $/ \mathrm{mL}$ e os linfócitos TDC4+ superiores a 500 céls $/ \mathrm{mm}^{3}$.

Alguns indivíduos não respondem ao tratamento de maneira efetiva devido à resistência de cepas virais, baixa adesão em função dos efeitos adversos ao tratamento, além de potenciais interações medicamentosas e alimentares capazes de refletir na falha terapêutica.

O uso contínuo de alguns medicamentos disponibilizados na TARV resulta em efeitos colaterais (náusea, enjôos, mal-estar) que estão relacionados à adesão do paciente ao tratamento (MASIKINI P, MPONDO B; SANTOS SLF et al., 2018).

Nas primeiras décadas da doença os déficits de vitaminas e minerais e a desnutrição energético-proteica (DEP) eram caracterizados como os maiores problemas nutricionais da época, e a desnutrição era responsável por $80 \%$ da mortalidade de PVHA, e essa situação foi revertida graças ao avanço da TARV (SILVA KGLSM et al., 2016).

De acordo com o último Boletim Epidemiológico HIV/Aids (2018), foram notificados 17.248 novos casos de infecção pelo HIV, sendo 4.197 na região Nordeste e 160 casos no estado do Piauí.

Diante dos dados epidemiológicos e dos impactos da infecção pelo HIV e sua possível progressão para Aids no estado nutricional e nos aspectos clínicos de PVHA em uso ou não de TARV, este estudo teve como objetivo descrever em formato de relatos de casos aspectos clínicos e nutricionais em pessoas que vivem com HIV/Aids atendidas em ambulatório de um hospital na capital do Piauí. 


\section{DETALHAMENTO DOS CASOS}

Para composição dos relatos de casos, foram considerados os seguintes critérios de inclusão: estar em tratamento em Teresina; ter idade maior que 20 anos; no momento, não fazer uso de suplementação de vitaminas ou minerais; não apresentar diagnóstico de doenças metabólicas (diabetes, síndrome metabólica, distúrbios da tireóide), doenças ósseas em tratamento, insuficiência renal crônica, hepatopatias, doenças gástricas ou síndromes disabsortivas, neoplasias; mulheres grávidas e lactantes).

Os dados clínicos foram obtidos tanto por meio de entrevista direta ao paciente (tempo de TARV e sintomas frequentes), como por busca nos prontuários do hospital (CD4, carga viral e terapia medicamentosa). O Índice de Massa Corpórea (IMC) foi obtido por meio da razão peso/estatura ${ }^{2}\left(\mathrm{Kg} / \mathrm{m}^{2}\right)$ para definir o estado nutricional de acordo com a OMS (2010), segundo a seguinte classificação para adultos: desnutrição $\left(<18,5 \mathrm{~kg} / \mathrm{m}^{2}\right)$, eutrofia $\left(18,5-24,9 \mathrm{Kg} / \mathrm{m}^{2}\right)$ e excesso de peso $\left(>25 \mathrm{Kg} / \mathrm{m}^{2}\right)$.

A massa corpórea foi mensurada, em triplicata, por meio de uma balança eletrônica plana Seca®e a altura por meio de um estadiômetro portátil Alturaexata®, com o qual posicionou o indivíduo localizando os pontos anatômicos (ocipital, costas, nádegas e panturrilhas). A circunferência da cintura e do pescoço foram mensuradas com uma fita métrica. O consumo alimentar foi coletado por meio do Recordatório de 24 horas de uma única tomada.

Todos os procedimentos realizados com os pacientes foram preservados, conforme preconizado nas "Diretrizes e Normas Regulamentadoras de Pesquisa Envolvendo Seres Humanos" do Conselho Nacional de Saúde ํㅜ66/2012 (BRASIL, 2012). O projeto foi cadastrado na Plataforma Brasil para ser avaliado pelo Comitê de Ética em Pesquisa da Universidade Federal do Piauí, sendo APROVADO (parecer 2.100.110 CEP/UFPI).

PACIENTE 1: A.R.M.S., 33 anos, sexo masculino, auxiliar de serviços gerais, referiu ter pele de cor parda, possui 9 anos de estudo, solteiro, renda de 1 salário mínimo. Nega ser fumante e ingere bebida alcoólica até três vezes por semana. Não possui doenças crônicas não transmissíveis. Iniciou a TARV há um mês utilizando Tenofovir + Lamivudina e Dolutegravir, referindo tosse e dispneia, além do uso de antibiótico no último mês. Sua carga viral era de 17.020 cópias e contagem de CD4 igual a $678 \mathrm{cé} / \mathrm{s} / \mathrm{mm}^{3}$, por meio do método de quantificação de RNA. A avaliação antropométrica demonstrou que o mesmo tem obesidade grau I (IMC= $\left.30,9 \mathrm{Kg} / \mathrm{m}^{2}\right)$, risco elevado de complicações relacionadas à obesidade $(\mathrm{CC}=109,8 \mathrm{~cm})$ e $\mathrm{CP}=41,5 \mathrm{~cm}$. Quanto a alimentação, o R24h demonstrou que a refeição do dia anterior desse indivíduo foi hipocalórica (512,38 Kcal).

PACIENTE 2: R.Z.O., 35 anos, sexo feminino, dona de casa, negra, 10 anos de estudo, solteira, renda de 1 salário mínimo. Não é tabagista e não ingere bebida alcoólica. Nega DCNT e possui três meses de TARV (Atazanavir, Tenovofir, Lamivudina, e Ritonavir), referindo perda de peso de $1 \mathrm{~kg}$. Desde o diagnóstico da doença, relata vômitos e constipação intestinal, e uso de dipirona no último mês. Exames laboratoriais apontam carga viral não detectada e CD4 igual a $65 \mathrm{céls} / \mathrm{mm}^{3}$. Segundo a antropometria, encontra-se com Magreza grau I $\left(\mathrm{IMC}=18,3 \mathrm{Kg} / \mathrm{m}^{2}\right)$, sem risco de complicações relacionadas à obesidade $(\mathrm{CC}=72 \mathrm{~cm})$ e $\mathrm{CP}=$ $33,5 \mathrm{~cm}$. O consumo de alimentos no dia anterior à entrevista revelou um porte calórico de 1.924,18 Kcal.

PACIENTE 3: F.S.S., 38 anos, sexo feminino, cozinheira, solteira, negra, com 5 anos de estudo, possui renda inferior a 1 salário mínimo. Refere tabagismo há 6 anos, consumindo pelo menos 1 cigarro ao dia e nega ingerir bebida alcoólica. Não possui outra patologia e iniciou a TARV há um mês, com o uso de Tenovofir, Lamivudina e Dolutegravir. Não referiu sintomas associados e ingeriu antibiótico nos últimos 30 dias. Detectou-se 128.928 cópias de carga viral e CD4= $426 \mathrm{céls} / \mathrm{mm}^{3}$. Apresenta-se eutrófica $\left(\mathrm{IMC}=20,9 \mathrm{Kg} / \mathrm{m}^{2}\right)$ e sem risco de complicações relacionadas à obesidade $(\mathrm{CC}=72 \mathrm{~cm}), \mathrm{CP}=30,5 \mathrm{~cm}$. Quanto à ingestão alimentar, seu R24h revelou consumo de 770,45 Kcal.

PACIENTE 4: J.T.C.F., 22 anos, sexo masculino, estudante, pardo, solteiro, 5 anos de estudo, refere renda inferior a um salário mínimo. Nega tabagismo e afirma ingerir bebida alcoólica raramente. Nega outra patologia e iniciou a TARV há 5 meses (Tenovofir, Lamivudina e Dolutegravir). 
Não foram detectadas cópias de carga viral $/ \mathrm{mL}$ e encontra-se com CD4= 836 céhls $/ \mathrm{mm}^{3}$. Encontra-se com sobrepeso $\left(\mathrm{IMC}=25,2 \mathrm{Kg} / \mathrm{m}^{2}\right)$ e sem risco de complicações relacionadas à obesidade $(C C=79 \mathrm{~cm})$ e $\mathrm{CP}=$ $37,9 \mathrm{~cm}$. Sua alimentação no dia anterior revelou um VCT de $2.643 \mathrm{Kcal}$.

PACIENTE 5: J.P.S., 50 anos, sexo masculino, desempregado, pardo, divorciado, 11 anos de estudo, possui renda inferior a 1 salário mínimo. Nega etilismo e tabagismo. Está sob tratamento antiretroviral há 10 meses, fazendo uso de Tenofovir, Lamivudina e Efafirenz, e afirma sentir dores abdominais. Não houve detecção de carga viral e o mesmo possui contagem de CD4 igual a 637 céls $/ \mathrm{mm}^{3}$. Encontra-se eutrófico $\left(\mathrm{IMC}=23,6 \mathrm{Kg} / \mathrm{m}^{2}\right)$ e não possui risco de complicações relacionadas à obesidade $(\mathrm{CC}=77 \mathrm{~cm})$ e $\mathrm{CP}=36,4$ $\mathrm{cm}$. No dia anterior à entrevista, o paciente consumiu 1.832,19 Kcal em alimentos.

PACIENTE 6: R.D.C.S., 22 anos, sexo masculino, vendedor, pardo, solteiro, com 11 anos de estudo, possui renda de um salário mínimo. Atualmente não fuma, porém refere suspensão do tabagismo há um ano, e ingere bebida alcoólica pelo menos 3 vezes durante a semana. Refere uma internação, faz TARV há 3 meses (Tenovofir, Lamivudina e Dolutegravir) e queixa-se de dores abdominais, tosse e falta de ar. Quanto à carga vira, detectou-se 117.501 cópias $/ \mathrm{mL}$ e contagem de CD4= $730 \mathrm{céls} / \mathrm{mm}^{3}$. Relata ingestão de antiinflamatório no último mês. Apresenta-se eutrófico $\left(\mathrm{IMC}=21,8 \mathrm{Kg} / \mathrm{m}^{2}\right)$ e sem risco de complicações relacionadas à obesidade $(\mathrm{CC}=81,4 \mathrm{~cm})$ e $\mathrm{CP}=37,7 \mathrm{~cm}$. O paciente consumiu $3.418,6 \mathrm{Kcal}$ no dia anterior ao R24h.

PACIENTE 7: M.C.R.A., 35 anos, sexo feminino, dona de casa, parda, 5 anos de estudo, solteira, com renda de um salário mínimo. Nega tabagismo, porém parou de fumar há 8 anos e nega alcoolismo. Relata 2 internações e 12 meses de TARV, com o uso de Tenovofir, Lamivudina e Dolutegravir. Não houve detecção de carga viral e a contagem de CD4 está igual a $354 \mathrm{céls} / \mathrm{mm}^{3}$. Encontra-se eutrófica $\left(I M C=18,9 \mathrm{Kg} / \mathrm{m}^{2}\right) \mathrm{e}$ sem risco de complicações relacionadas à obesidade $(C C=71,8 \mathrm{~cm})$ e $C P=30,5 \mathrm{~cm}$. A entrevista por meio do $\mathrm{R} 24 \mathrm{~h}$ revelou que sua ingestão de alimentos foi equivalente a $1.131 \mathrm{Kcal}$ no dia anterior.

PACIENTE 8: A.L.L.S., 49 anos, sexo feminino, técnica de enfermagem, parda, divorciada, com 11 anos de estudo e renda de aproximadamente 4 salários mínimos. Nega etilismo e tabagismo. Também nega patologias, internações, uso de medicamentos e perda de peso. No momento, a mesma não fazia uso de TARV. Não apresentou detecção de carga viral e CD4= 515 céls $/ \mathrm{mm}^{3}$. Encontra-se com sobrepeso (IMC= $\left.25,5 \mathrm{Kg} / \mathrm{m}^{2}\right)$ e com risco elevado de complicações relacionadas à obesidade $(\mathrm{CC}=83,8 \mathrm{~cm})$ e $\mathrm{CP}=31,5 \mathrm{~cm}$. Seu consumo de alimentos do dia anterior foi de 1.287,44 Kcal.

O quadro 1 resume os principais dados biológicos, clínicos e nutricionais dos casos em estudo.

Quadro 1 - Características biológicas, clínicas e nutricionais de PVHA em uso ou não de TARV. Teresina, 2019.

\begin{tabular}{|c|c|c|c|c|c|c|c|c|c|}
\hline \multirow[b]{2}{*}{ Caso } & \multicolumn{2}{|c|}{ Biológicos } & \multicolumn{3}{|c|}{ Clínicos } & \multicolumn{4}{|c|}{ Nutricionais } \\
\hline & $\begin{array}{l}\text { Idade } \\
\text { (Anos) }\end{array}$ & Sexo & TARV* & $\begin{array}{c}\text { CD4 } \\
\text { (céls } / \mathbf{m m}^{3} \text { ) }\end{array}$ & $\begin{array}{l}\text { Carga Viral } \\
\text { (Cópias } / \mathrm{mL} \text { ) }\end{array}$ & $\begin{array}{c}\text { IMC } \\
\left(\mathrm{Kg} / \mathrm{m}^{2}\right)\end{array}$ & $\begin{array}{l}\mathrm{CC} \\
(\mathrm{cm})\end{array}$ & $\begin{array}{l}\mathrm{CP} \\
(\mathrm{cm})\end{array}$ & $\begin{array}{l}\text { VCT } \\
\text { (Kcal) }\end{array}$ \\
\hline 1 & 33 & $M$ & $3 T C+T D F+D T G$ & 678 & 17.020 & 30,9 & 109,8 & 41,5 & 512,38 \\
\hline 2 & 35 & $\mathrm{~F}$ & ATV/r+TDF+3TC+RTV & 65 & Não detec & 18,3 & 72 & 33,5 & $1.924,18$ \\
\hline 3 & 38 & $\mathrm{~F}$ & $3 T C+T D F+D T G$ & 426 & 128.928 & 20,9 & 72 & 30,5 & 770,45 \\
\hline 4 & 22 & $M$ & $3 T C+T D F+D T G$ & 836 & Não detec & 25,2 & 79 & 37,9 & $2.643,0$ \\
\hline 5 & 50 & $M$ & $3 T C+T D F+E F V$ & 637 & Não detec & 23,6 & 77 & 36,4 & $1.832,19$ \\
\hline 6 & 22 & $\mathrm{M}$ & 3TC+TDF+DTG & 730 & 117.501 & 21,8 & 81,4 & 37,7 & $3.418,6$ \\
\hline 7 & 35 & $\mathrm{~F}$ & 3TC+TDF+DTG & 354 & Não detec & 18,9 & 71,8 & 30,5 & $1.131,0$ \\
\hline 8 & 49 & $\mathrm{~F}$ & & 515 & Não detec & 25,5 & 83,8 & 31,5 & $1.287,44$ \\
\hline
\end{tabular}

Fonte: Pesquisa direta, 2019.

*Legenda: M (masculino); F (feminino); TARV (terapia antiretroviral); Lamivudina (3TC); Tenofovir (TDF); Dolutegravir (DTG); Atazanavir (ATV/r); Efavirenz (EFV); IMC (índice de massa corporal); CC (circunferência da cintura); CP (circunferência do pescoço); VCT (valor calórico total). 


\section{DISCUSSÃO}

Nos relatos descritos acima, predominou-se o uso de TARV, incluindo Tenofovir e Lamivudina, que são Inibidores Nucleosídeos/ Nucleotídeos da Transcriptase Reversa, que têm como alvo a proteína transcriptase reversa, sendo está a classe de fármacos antirretrovirais considerada primordial no tratamento, normalmente administrado em um único comprimido combinado com outros agentes farmacológicos. Já o Dolutegravir (Inibidor da Integrase) é recomendado em casos de falha virológica ou quando há resistência a outras classes de antirretrovirais, que pode ser detectada por meio de genotipagem realizada nos 12 meses anteriores (ALCORN K, et al., 2013; BRASIL, 2016).

Quanto aos demais antirretrovirais, pouco citados nos casos, Atazanavir e Ritonavir, que são Inibidores de Protease, que atuam na inibição da enzima protease, evitando a produção de células infectadas pelo HIV. Já o Efavirenz faz parte da classe dos Inibidores não-nucleosídeos da transcriptase reversa, que atuam na inibição da mobilidade e flexibilidade do sítio ativo responsável pela estrutura do DNA, o que resulta no declínio da atividade da enzima transcriptase reversa (BRASIL, 2013; COSTA CCP, 2015).

Segundo Figueiredo LA, et al. (2014), os medicamentos utilizados na TARV proporcionam efeitos colaterais incômodos aos usuários, tais como náuseas, vômitos, diarreia, elevação dos níveis de colesterol e triglicérides séricos e as lipodistrofias, sendo essa uma das causas da falta de adesão ao tratamento por alguns pacientes.

Nesse relato de casos, os sintomas mais citados foram no trato gastrintestinal: constipação intestinal e dor abdominal. Segundo Santos ASAC, et al. (2017), o principal alvo do vírus é o sistema imune intestinal, comprometendo sua estrutura e função, possibilitando um avanço na progressão da doença. Porém, mesmo com o uso da TARV, alguns sintomas gastrintestinais podem ocorrer, sendo este um fator importante para a diminuição da adesão e descontinuidade do tratamento.

A maior parte dos pacientes da série de casos apresentou contagem de células T CD4+ entre 200 e 500 céls $/ \mathrm{mm}^{3}$, que de acordo com Brasil (2002), estão num estágio da infecção causado por sinais e sintomas de menor impacto, mas muitos podem manter-se assintomáticos. Outros pacientes encontram-se em baixo risco de desenvolver doenças (CD4 $>500$ céls $/ \mathrm{mm}^{3}$ ). Apenas um indivíduo apresentou contagem de CD4 entre 50 e 200 céls $/ \mathrm{mm}^{3}$, o que sugere alto risco de surgimento de doenças oportunistas.

A análise da ingestão de alimentos revelou que a maioria dos entrevistados consumiu dietas com um valor calórico total (VCT) abaixo do que é recomendado pelo Ministério da Saúde (2008) - 2.000 Kcal - no dia anterior à entrevista. Porém, vale ressaltar que a aplicação do R24h em uma única entrevista mensura a ingestão alimentar de um indivíduo esporadicamente, sendo considerado infiel em relação à aplicação de um questionário que avalia o consumo de alimentos durante a semana.

Sabe-se que o cuidado clínico e nutricional de PVHA, em conjunto, são capazes não apenas de aumentar a expectativa, como também melhorar a qualidade de vida dos mesmos. De acordo com Paula EP et al. (2010), os cuidados adequados com a alimentação e nutrição desses indivíduos são primordiais para a manter o sistema imunológico saudável, porém, um conjunto de fatores podem levar uma PVHA a ter dificuldades em garantir uma boa nutrição, fatores estes relacionados não apenas à infecção pelo HIV, mas também o uso contínuo da TARV.

Quanto ao estado nutricional, a maioria das PVHA estudadas encontravam-se eutrófica (4); 3 estavam com sobrepeso ou obesidade e apenas 1 apresenta baixo peso. Estudos atuais mostram que graças ao avanço da TARV, a incidência de baixo peso e desnutrição declinou significativamente nas últimas décadas. Mesmo que o ganho de peso seja benéfico ao sistema imune, houve aumento no surgimento de doenças crônicas não transmissíveis e comorbidades associados ao uso da terapia antirretroviral. $O$ excesso de gordura em pessoas que vivem com HIV/Aids configura-se como um fator adicional para resistência insulínica, diabetes mellitus e síndrome metabólica (GUIMARÃES NS, 2016).

No que diz respeito à Circunferência do Pescoço (CP), tais relatos apontam dois casos (paciente 1 e 4 ) em que tanto a CP quanto o IMC indicam acúmulo de gordura e sobrepeso, respectivamente, sendo que em 
um destes casos (paciente 1), a CC aponta risco elevado de complicações metabólicas relacionadas à obesidade. Estudos recentes mostram a relação entre a circunferência do pescoço (CP) e outros parâmetros antropométricos - IMC, CC e CQ - como indicadores de adiposidade em crianças, adultos e idosos. Além disso, a CP também é apontada como medida direta ligada à aterosclerose em PVHA. Ressalta-se que o acúmulo de gordura nas regiões do pescoço e da cintura pode, também, ser indício de lipodistrofia, comumente observada em PVHA em uso de TARV (OLIVEIRA NA, et al., 2019; DERESZ LF et al., 2018).

\section{CONSIDERAÇÕES FINAIS}

Visto que uma alimentação e nutrição adequadas, unidas à TARV, são capazes de proporcionar tanto uma maior expectativa quanto uma melhora na qualidade de vida de PVHA, tais relatos de casos mostraram a importância de descrever não apenas os aspectos clínicos, mas também os aspectos nutricionais, para que haja um acompanhamento mais detalhado e individualizado para tal população estudada. Ressalta-se também a importância de realizar mais estudos englobando aspectos nutricionais em PVHA.

\section{REFERÊNCIAS}

1. ALCORN K, et al. Medicamentos Antirretrovirais, 2ed - traduzida. Aidsmap, 2013; 70p.

2. BRASIL, Ministério da Saúde. Secretaria de Vigilância em Saúde. Departamento de Vigilância Epidemiológica. Guia de bolso para doenças infecciosas e parasitárias, 8ed. Brasília, 2013a; 448p.

3. BRASIL, Ministério da Saúde. Secretaria de Vigilância em Saúde. Departamento de DST, Aids e Hepatites Virais. Protocolos clínicos e diretrizes terapêuticas para manejo da infecção pelo HIV em adultos, 1ed. Brasília, 2013b; 412p. Disponível em: http://www.aids.gov.br/pt-br/pub/2013/protocolo-clinico-e-diretrizes-terapeuticas-para-manejo-da-infeccaopelo-hiv-em-adultos Acesso em: 21 mai 2019

4. BRASIL, Ministério da Saúde. Secretaria de Ciência, Tecnologia e Insumos Estratégicos. Conselho Nacional de Incorporação de Tecnologia do SUS. Ampliação de uso dos medicamentos antirretrovirais dolutegravir (DTG) e darunavir (DRV). Relatório de Recomendação, no 227. Brasília, 2016. Disponível em: http://conitec.gov.br/images/Relatorios/2016/Relatorio-DolutegravirDarunavir-final-Republicacao.pdf Acesso em: 21 mai 2019.

5. BRASIL. Ministério da Saúde. Secretaria de Vigilância em Saúde - Boletim Epidemiológico HIV/Aids. Brasília, 2018.

6. BRASIL. Ministério Da Saúde. Instituto Nacional do Seguro Social. Norma técnica de avaliação da incapacidade laborativa para fins de benefícios previdenciários em HIV/AIDS. Resolução INSS/DC n. 089, 05 abril 2002. Brasília; 2002.

7. COSTA CCP, et al. O Efavirenz: Relação estrutura-atividade e métodos de síntese. Revista Virtual de Química, 2015; 7(4): 1347-1370.

8. DERESZ LF, et al. Consumo Alimentar e Risco Cardiovascular em pessoas vivendo com HIV/Aids. Ciência e Saúde Coletiva, 2018; 23(8): 2533-2542.

9. FIGUEIREDO LA, et al. Oferta de ações e serviços de saúde para o manejo do HIV/Aids, sob a perspectiva dos usuários. Revista da escola enfermagem da USP, 2014; 48(6).

10. GUIMARÃES NS, et al. Prevalência de excesso de peso, obesidade central e risco de comorbidades metabólicas em adultos com HIV/Aids sem terapia antirretroviral. Revista Brasileira de Promoção à Saúde, 2016; 29(3).

11. MASIKINI P, MPONDO B. HIV drug resistance mutations following poor adherence in HIV-infected patient: a case report. Clinical Case Report, 2015; 3(6): 676-681.

12. OLIVEIRA NA, et al. A medida da circunferência do pescoço pode ser usada como indicador de adiposidade corporal? Revisão Sistemática. Revista Brasileira de Obesidade, Nutrição e Emagrecimento, 2019; 13(77): 157-165.

13. Organização Mundial de Saúde (OMS). Folha Informativa - HIV/Aids, Banco de Notícias. Nov 2017. Disponível em: $<$ https://www.paho.org/bra/index.php?option=com_content\&view=article\&id=5666:folha-informativa-hiv-aids\&ltemid=812> Acesso em: 19/07/2018.

14. PAULA EP, et al. Considerações nutricionais para adultos com HIV/Aids. Revista Matogrossense de Enfermagem, 2010; 1(2): 148-165.

15. SANTOS ASAC, et al. Effectiveness of nutritional treatment and synbiotic use on gastrointestinal symptoms reduction in HIV-infected patients: Randomized clinical Trial. Clinical Nutrition, 2017; 36, 680-685.

16. SANTOS SLF. Fatores desfavoráveis na adesão à terapia antirretroviral e diagnóstico sorológico: revisão da literatura. Revista Expressão Católica Saúde, 2018; 3(1).

17. SILVA KGLSM, et al. Perfil clínico-nutricional de portadores do vírus HIV atendidos em um hospital de referência do Nordeste brasileiro. Jornal brasileiro de doenças sexualmente transmissíveis, 2016; 28 (2): p.50-55. 